\title{
Timed-Release Hierarchical Identity-Based Encryption
}

\author{
Toru Oshikiri \\ Graduate School of Engineering \\ Tokyo Denki University \\ Tokyo, Japan
}

\author{
Taiichi Saito \\ Tokyo Denki University \\ Tokyo, Japan
}

\begin{abstract}
We propose a notion of hierarchical identity-based encryption (HIBE) scheme with timed-release encryption (TRE) mechanism, timed-release hierarchical identity-based encryption (TRHIBE), and define its security models. We also show a generic construction of TRHIBE from HIBE and one-time signature, and discuss the security of the constructed scheme.
\end{abstract}

Keywords-timed-release encryption, hierarchical identity-based encryption, one-time signature

\section{INTRODUCTION}

Timed-release encryption (TRE) [1] [2] [3] [4] [5] is an encryption mechanism that allows a receiver to decrypt a ciphertext only after the time that a sender designates.

Timed-release identity-based encryption (TRIBE) [6] is an extension of TRE having a function of identity-based encryption (IBE). In TRIBE, even a legitimate receiver cannot decrypt a ciphertext using secret key until the time designated by the sender. A TRIBE system consists of a key generation center (KGC), a time server (TS), senders and receivers. A sender encrypts a message using an identity of a receiver and a time after which the ciphertext could be decrypted. The KGC generates a secret key corresponding to an identity of a receiver. The TS periodically broadcasts a time signal corresponding to the current time. The receiver decrypts the ciphertext using the secret key and the time signal corresponding to the time designated by the sender. TRIBE systems use identity of user as his/her public key. TRIBE has an advantage that it does not require linking public keys to identity such as PKI.

Timed-release hierarchical identity-based encryption (TRHIBE) is another extension of TRE having a function of hierarchical identity-based encryption (HIBE). In TRHIBE, even a legitimate receiver cannot decrypt a ciphertext using secret key until a time designated by a sender. A TRHIBE system consists of senders, multiple KGCs, a single TS, and receivers. The KGCs and users have a hierarchical structure in which each KGC generates a secret key corresponding to an identity of a child KGC or a child user. Therefore, the load of derivation of users secret keys can be distributed to multiple KGCs. A sender encrypts a message using an identity of a receiver and a time. The TS periodically broadcasts a time signal corresponding to the current time. The receiver decrypts the ciphertext using the secret key and the time signal corresponding to the time designated by the sender.

\section{RELATED WORKS}

In TRIBE, a user can decrypt a ciphertext only when the user has the receiver's secret key and the time signal generated by TS. Then, if the receiver does not have the time signal or the TS does not have the secret key, they cannot decrypt the ciphertext. In [6], two security models of TRIBE are defined. One is security against malicious receiver, IND-ID-CCA $A_{C R}$ security. The other is security against malicious TS, INDID-CCA $A_{\text {TS }}$ security. A generic construction of TRIBE that achieves the security is also shown in [6]. It is a combination of two IBE schemes and a one-time signature scheme, based on "Parallel Encryption" by Dodis-Katz [7], and the security is proved in the standard model.

\section{Contribution}

In this paper, we introduce timed-release hierarchical identity-based encryption (TRHIBE) and define two security models. One is security against malicious receiver, IND-hID$\mathrm{CCA}_{\mathrm{CR}}$ security. The other is security against malicious TS, IND-hID-CCA TS security. We also present a generic construction of TRHIBE. It is a combination of two HIBE schemes and a one-time signature scheme, also based on "Parallel Encryption". We see that if the primitive HIBE schemes are IND-hIDCCA secure and the primitive one-time signature scheme is OT-sEUF-CMA secure, then the constructed TRHIBE scheme is IND-hID-CCA $A_{C R}$ secure and IND-hID-CCA ${ }_{T S}$ secure in the standard model.

\section{PReliminaries}

In this section, we review hierarchical identity-based encryption (HIBE) and one-time signature, which we use later.

\section{A. Hierarchical Identity-Based Encryption}

In an HIBE scheme, the single KGC functionality of generating secret keys is divided into partial ones and they are delegated to multiple KGCs. If a KGC is assigned an identity vector, $\mathrm{ID}^{(k-1)}=\left(I_{1}, \ldots, I_{k-1}\right)$, and given a secret key, $d_{\mathrm{ID}^{(k-1)}}$, corresponding to the identity vector, then it can generate a secret key, $d_{\mathrm{ID}^{(k)}}$, corresponding to an identity vector, $\mathrm{ID}^{(k)}=\left(I_{1}, \ldots, I_{k-1}, I_{k}\right)$. We may denote an identity by ID if we need not to specify its hierarchy depth. 
Let $\lambda$ be a security parameter and $\ell$ be a maximum depth of hierarchy. An hierarchical identity-based encryption scheme $\mathcal{H} \mathcal{I B E}$ consists of five probabilistic polynomialtime algorithms $\mathcal{H} \mathcal{I B E}=($ HIBE.Setup, HIBE.Ext, HIBE.Del, HIBE.Enc, HIBE.Dec). The setup algorithm HIBE.Setup takes $\lambda$ and $\ell$ as input, and outputs a public parameter params and a master secret key $m s k$. The extract algorithm HIBE.Ext takes params, msk, and an identity $\mathrm{ID}^{(k)}=\left(\mathrm{I}_{1}, \ldots, \mathrm{I}_{k}\right)$ as inputs, and outputs a decryption key $d_{\mathrm{ID}^{(k)}}$. The delegate algorithm HIBE.Del takes params, ID ${ }^{(k)}, d_{\mathrm{ID}^{(k)}}$ and an identity $\mathrm{ID}^{(k+1)}$ as inputs, and outputs a decryption key $d_{\mathrm{ID}}{ }^{(k+1)}$. The encryption algorithm HIBE.Enc takes params, ID, a message $m$ as inputs, and outputs a ciphertext $c$. The decryption algorithm HIBE.Dec takes params, a ciphertext $c$ and a decryption key $d_{\mathrm{ID}}$ as inputs, and outputs the plaintext $m^{\prime}$ or $\perp$. These algorithms are assumed to satisfy that if (params, msk) = $\operatorname{HIBE} . \operatorname{Setup}(\lambda)$ and $d_{\mathrm{ID}}=\operatorname{HIBE} . \operatorname{Ext}($ params, msk, ID) or $d_{\mathrm{ID}(k)}=$ HIBE.Del $\left(\right.$ params $\left., \mathrm{ID}^{(k-1)}, d_{\mathrm{ID}^{(k-1)}}, \mathrm{ID}^{(k)}\right)$ for $k \leq$ $n$, then HIBE.Dec $\left(\right.$ params, $d_{\mathrm{ID}}$, HIBE.Enc $($ params, ID,$\left.m)\right)=$ $m$ for any $m$.

1) IND-hID-CCA Security: We review a standard security notion for HIBE: indistinguishability against adaptive hierarchical identity and chosen ciphertext attacks (IND-hID-CCA) security [8] [9]. We here describe the IND-hID-CCA security for HIBE scheme $\mathcal{H} \mathcal{I B E}$ based on the following IND-hIDCCA game between a challenger $\mathcal{C}$ and an adversary $\mathcal{A}$.

Setup

Phase1

$\mathcal{C}$ runs (params, msk $) \leftarrow \operatorname{HIBE} \cdot \operatorname{Setup}(\lambda, \ell) . \mathcal{C}$ sends params to $\mathcal{A}$ and keeps msk secret.

$\mathcal{A}$ can adaptively issue extraction queries ID and decryption queries $(\mathrm{ID}, c)$. $\mathcal{C}$ responds to an extraction query ID by running $d_{\mathrm{ID}_{j}}=$ HIBE.Ext (params, msk, ID) and returning $d_{\mathrm{ID}}$ to $\mathcal{A}$. $\mathcal{C}$ responds to a decryption query $(\mathrm{ID}, c)$ by running $d_{\mathrm{ID}}=\mathrm{HIBE}$.Ext (params, msk, ID) and Challenge $m^{\prime}=\mathrm{HIBE} . \operatorname{Dec}\left(d_{\mathrm{ID}}, c\right)$, and returning $m^{\prime}$ to $\mathcal{A}$.

$\mathcal{A}$ sends two messages $m_{0}, m_{1}$ such that $\left|m_{0}\right|=$ $\left|m_{1}\right|$, and an identity to be challenged $\mathrm{ID}^{*}$ to $\mathcal{C}$. The challenge identity $\mathrm{ID}^{*}$ must differ from any ID issued as extraction query in Phase1, and any its prefixes. $\mathcal{C}$ randomly chooses $b \in$ $\{0,1\}$ and sends a challenge ciphertext $c^{*}=$ HIBE.Enc $\left(\right.$ params, ID* $\left.m_{b}\right)$ to $\mathcal{A}$.

Phase2

$\mathcal{A}$ can adaptively issue extraction queries ID and decryption queries (ID, $c$ ) in the same way as in Phase1 except that the extraction queries ID must differ from the challenge identity ID* and its prefixes, and decryption queries (ID, $c$ ) must differ from the pair $\left(\mathrm{ID}^{*}, c^{*}\right)$.

Guess

$\mathcal{A}$ outputs a guess $b^{\prime} \in\{0,1\}$ and wins if $b=b^{\prime}$.

We define an advantage of $\mathcal{A}$ in the IND-hID-CCA game as $A d v_{\mathcal{H} \mathcal{I} \mathcal{B}, A}^{\text {INDhID-CCA }}(\lambda)=\left|2 \operatorname{Pr}\left[b=b^{\prime}\right]-1\right|$, in which the probability is taken over the random coins used by $\mathcal{C}$ and $\mathcal{A}$. We say that the HIBE scheme $\mathcal{H} \mathcal{I B E}$ is IND-hID-CCA secure if, for any probabilistic polynomial-time adversary $\mathcal{A}$, the function $A d v_{\mathcal{H} \mathcal{I} \mathcal{B}, \mathcal{A}}^{\mathrm{IND}-\mathrm{hI}-\mathrm{CCA}}(\lambda)$ is negligible in $\lambda$.

\section{B. Signature}

Let $\lambda$ be a security parameter. $\mathcal{A}$ signature scheme $\mathcal{S I G}$ consists of three probabilistic polynomial-time algorithms $\mathcal{S I G}=$ (SigGen, Sign, Verify). The key generation algorithm SigGen takes $\lambda$ as input, and outputs a signing key $s k$ and a verification key $v k$. The signing algorithm Sign takes $s k$ and a message $m$ as inputs, and outputs a signature $\sigma$. The verification algorithm Verify takes $v k$, a message $m$, and a signature $\sigma$ as inputs, and outputs accept or reject. These algorithms are assumed to satisfy that if $(s k, v k)=\operatorname{SigGen}(\lambda)$ then $\operatorname{Verify}(v k, m, \operatorname{Sign}(s k, m))=$ accept for any $m$.

1) OT-sEUF-CMA Security: We review a security notion for one-time signature scheme: one-time strong existential unforgeability against chosen message attacks (OT-sEUF-CMA) security [10]. We here describe the OT-sEUF-CMA security for signature scheme $\mathcal{S I G}$ based on the following OT-sEUFCMA game between a challenger $\mathcal{C}$ and an adversary $\mathcal{A}$.

Setup

$\mathcal{C}$ runs the $(s k, v k) \leftarrow \operatorname{Sig} \operatorname{Gen}(\lambda) . \mathcal{C}$ sends $v k$ to $\mathcal{A}$ and keeps $s k$ secret.

Query

$\mathcal{A}$ can issue a signing query $m$ to $\mathcal{C}$ only once. $\mathcal{C}$ responds to the singing query $m$ by running $\sigma=\operatorname{Sign}(v k, m)$ and returning $\sigma$ to $\mathcal{A}$.

\section{Forge}

$\mathcal{A}$ outputs a pair $\left(m^{*}, \sigma^{*}\right)$.

We define the advantage of $\mathcal{A}$ in the OT-sEUF-CMA game as $\operatorname{Adv}_{\mathcal{S} \mathcal{I} \mathcal{G}, \mathcal{A}}^{\mathrm{OT}-\mathrm{CMA}}(\lambda)=\operatorname{Pr}\left[\operatorname{Verify}\left(v k, m^{*}, \sigma^{*}\right)=\right.$ accept $\wedge$ $\left.(m, \sigma) \neq\left(m^{*}, \sigma^{*}\right)\right]$, in which the probability is taken over the random coins used by $\mathcal{C}$ and $\mathcal{A}$. We say that the signature scheme $\mathcal{S I G}$ is OT-sEUF-CMA secure if, for any probabilistic polynomial-time adversary $\mathcal{A}$, the function $A d v_{\mathcal{S} \mathcal{I} \mathcal{O}, \mathcal{A}}^{\mathrm{TT}}{ }^{\mathrm{P} E \mathrm{CMA}}(\lambda)$ is negligible in $\lambda$.

\section{TIMED-RELEASE HiERARCHICAL IDENTITY-BASED ENCRYPTION(TRHIBE)}

In this section, we introduce timed-release hierarchical identity-based encryption(TRHIBE) scheme and define its security models.

A TRHIBE system consists of a single TS, multiple KGCs and multiple users connected through a communication network. The time server periodically broadcasts a time signal corresponding to the current time, and all users can receive the time signal. The single KGC functionality of generating secret keys is divided into partial ones and they are delegated to multiple KGCs. If a $\mathrm{KGC}$ is assigned an identity vector, $\mathrm{ID}^{(k-1)}=\left(I_{1}, \ldots, I_{k-1}\right)$, and given a secret key, $d_{\mathrm{ID}^{(k-1)}}$, corresponding to the identity vector, then it can generate a secret key, $d_{\mathrm{ID}^{(k)}}$, corresponding to an identity vector, $\mathrm{ID}^{(k)}=$ $\left(I_{1}, \ldots, I_{k-1}, I_{k}\right)$. We may denote an identity by ID if we need not to specify its hierarchy depth. A user (sender) encrypts a plaintext, designating another user (receiver) who can decrypt the ciphertext and a time only after which the ciphertext can be decrypted. The receiver can decrypt the ciphertext with the 
secret key that he/she has and the time signal that the time server broadcasts at the designated time.

Let $\lambda$ be a security parameter and $\ell$ be a maximum depth of system. An timed-release hierarchical identitybased encryption scheme $\mathcal{T} \mathcal{R} \mathcal{H} \mathcal{I B E}$ consists of seven probabilistic polynomial-time algorithms $\mathcal{T} \mathcal{R} \mathcal{H} \mathcal{I} \mathcal{E}=(T S$ Setup, KGC_Setup, Release, Extract, Delegate, Encrypt, Decrypt). The time server's setup algorithm TS_Setup takes $\lambda$ as input, and outputs a public key tpk and the corresponding secret key tsk. The key generation center's setup algorithm KGC_Setup takes $\lambda$ and the depth $\ell$ as input, and outputs a public parameter params and a master secret key msk. The release algorithm Release takes $t p k$, $t s k$ and a time period $T$ as inputs, and outputs a time signal $d_{T}$. The extract algorithm Extract takes params, msk, and an identity $\mathrm{ID}^{(k)}=\left(\mathrm{I}_{1}, \ldots, \mathrm{I}_{k}\right)$ as inputs, and outputs a decryption key $d_{\mathrm{ID}(k)}$. The delegate algorithm Delegate takes params, $\mathrm{ID}^{(k)}, d_{\mathrm{ID}^{(k)}}$ and an identity $\mathrm{ID}^{(k+1)}$ as inputs, and outputs a decryption key $d_{\mathrm{ID}}^{(k+1)}$. The encryption algorithm Encrypt takes tpk,params, $T$, and ID, and a message $m$ as inputs, and outputs a ciphertext $c$. The decryption algorithm Decrypt takes as inputs $t p k$, params, a ciphertext $c^{\prime}, d_{T}$, a user's secret key $d_{\mathrm{ID}}$, and outputs the plaintext $m^{\prime}$ or $\perp$. These algorithms are assumed to satisfy that Decrypt $\left(t p k\right.$, params, $d_{T}, d_{\mathrm{ID}}$, Encrypt $(t p k$, params, $T$, ID,$m))=m$ holds for any $m$, if $(t p k$, $t s k)=$ TS_Setup $(\lambda),($ params,$m s k)=\operatorname{KGC}$ Setup $(\lambda, \ell), s_{T}$ $=$ TR.Release $(t p k, t s k, T)$, and $d_{\mathrm{ID}}=$ HIBE.Ext $($ params, $m s k$, ID) hold, and that $d_{\mathrm{ID}^{(n)}}=\mathrm{HIBE}$.Ext $\left(\right.$ params, $\left.m s k, \mathrm{ID}^{(n)}\right)$ and $d_{\mathrm{ID}(k)}=\operatorname{HIBE} . \operatorname{Del}\left(\right.$ params, $\left.\mathrm{ID}^{(k-1)}, d_{\mathrm{ID}^{(k-1)}}, \mathrm{ID}^{(k)}\right)$ for $k \leq n$ hold.

\section{A. Security}

We can consider security against malicious TS and security against malicious receiver.

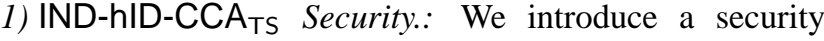
notion for TRHIBE: indistinguishability against adaptive hierarchical identity and chosen ciphertext attacks by timeservers (IND-hID-CCA $\mathrm{TS}_{\mathrm{TS}}$ ) security. This security ensures that a malicious time server, who has a secret key tsk, cannot obtain any information of message from ciphertext without decryption key $d_{\text {ID }}$. We here describe the IND-hID-CCA $A_{T S}$ security for a TRHIBE scheme $\mathcal{T} \mathcal{R} \mathcal{H} \mathcal{I} \mathcal{B}$ based on the following IND-hID$\mathrm{CCA}_{\mathrm{TS}}$ game between a challenger $\mathcal{C}$ and adversary $\mathcal{A}$.

\section{Setup}

$\mathcal{C}$ runs $($ tpk, tsk $) \leftarrow$ TS_Setup $(\lambda)$ and (params, $m s k) \leftarrow \mathrm{KGC} \operatorname{Setup}(\lambda, \ell) . \mathcal{C}$ sends $t p k, t s k$ and Phase1 params to $\mathcal{A}$ and keeps msk secret.

$\mathcal{A}$ can adaptively issue extraction queries ID and decryption queries $(T, \mathrm{ID}, c) . \mathcal{C}$ responds to an extraction query ID by running $d_{\mathrm{ID}}=$ Extract (params, msk, ID) and returning $d_{\mathrm{ID}}$ to $\mathcal{A}$. $\mathcal{C}$ responds to a decryption query $(T, \mathrm{ID}, c)$ by running $d_{T}=\operatorname{Release}(t p k, t s k, T), d_{\mathrm{ID}}=$ Extract (params, msk, ID) and $c=\operatorname{Decrypt}\left(d_{T}\right.$, $\left.d_{\mathrm{ID}}, c\right)$, and returning $c$ to $\mathcal{A}$.

\section{Challenge}

$\mathcal{A}$ sends two messages $m_{0}, m_{1}$ such that $\left|m_{0}\right|=$ $\left|m_{1}\right|$, a time period $T^{*}$ and an identity to be challenged ID* to $\mathcal{C}$. The challenge identity ID* must differ from any ID issued as extraction queries in Phase1 and any its prefixes. $\mathcal{C}$ randomly chooses $b \in\{0,1\}$ and sends a challenge ciphertext $c^{*}=\operatorname{Encrypt}\left(t p k\right.$, params, $\left.T^{*}, \mathrm{ID}^{*}, m_{b}\right)$ to $\mathcal{A}$.

Phase2

$\mathcal{A}$ can adaptively issue extraction queries ID and decryption queries $(T, I D, c)$ in the same way as Phase1 except that the extraction queries ID must differ from the challenge identity $I^{*}$ and its prefixes, and the decryption queries $(T, \mathrm{ID}, c)$ must differ from the tuple $\left(T^{*}, \mathrm{ID}^{*}, c^{*}\right)$.

Guess

$\mathcal{A}$ outputs a guess $b^{\prime} \in\{0,1\}$ and wins if $b=b^{\prime}$.

We define an advantage of $\mathcal{A}$ in the IND-hID-CCA TS $_{\text {game }}$ as $A d v_{\mathcal{T} \mathcal{R} \mathcal{H} \mathcal{I} \mathcal{B} \mathcal{A}_{\mathcal{A}}}^{\mathrm{IND}}(\lambda)=\left|2 \operatorname{Pr}\left[b=b^{\prime}\right]-1\right|$, in which the probability is taken over the random coins used by $\mathcal{C}$ and $\mathcal{A}$. We say that the TRIBE scheme $\mathcal{T} \mathcal{R} \mathcal{H} \mathcal{I} \mathcal{E}$ is IND-hID-CCA secure if, for any probabilistic polynomial-time adversary $\mathcal{A}$, the function $A d v_{\mathcal{T} \mathcal{R} \mathcal{H} \mathcal{I} \mathcal{B E}, \mathcal{A}}^{\text {IND-hIS }}(\lambda)$ is negligible in $\lambda$.

2) IND-hID-CCA $A_{C R}$ Security.: We introduce another security notion for TRIBE: indistinguishability against adaptive hierarchical identity and chosen ciphertext attacks by curious receiver (IND-hID-CCA ${ }_{C R}$ ) security. This security ensures that a receiver who has a decryption key $d_{\mathrm{ID}}$ cannot obtain any information of message from ciphertext without time signal $d_{T}$. We here describe the IND-hID-CCA $A_{C R}$ security for a TRIBE scheme $\mathcal{T} \mathcal{R} \mathcal{H} \mathcal{I B E}$ based on the following IND-hID$\mathrm{CCA}_{\mathrm{CR}}$ game between a challenger $\mathcal{C}$ and an adversary $\mathcal{A}$.

Setup

Phase1

$\mathcal{C}$ runs $(t p k, t s k) \leftarrow$ TS_Setup $(\lambda)$ and (params $m s k) \leftarrow$ KGC_Setup $(\lambda, \bar{\ell})$. $\mathcal{C}$ sends params, msk and $t p k$ to $\mathcal{A}$ and keeps tsk secret.

$\mathcal{A}$ can adaptively issue release queries $T$ and decryption queries $(\mathrm{T}, \mathrm{ID}, c)$. $\mathcal{C}$ responds to a release query $T$ by running $d_{T}=\operatorname{Release}(t p k, t s k, T)$ and returning $d_{T}$ to $\mathcal{A}$. $\mathcal{C}$ responds to a decryption query $(T, \mathrm{ID}, c)$ by running $d_{T}=\operatorname{Release}(t p k$, tsk, $T), d_{\mathrm{ID}}=\operatorname{Extract}($ paramsmsk, ID) and $c=\operatorname{Decrypt}\left(d_{T}, d_{\mathrm{ID}}, c\right)$, and returning $c$ to $\mathcal{A}$.

Challenge

$\mathcal{A}$ sends two messages $m_{0}, m_{1}$ such that $\left|m_{0}\right|=$ $\left|m_{1}\right|$, a time period $T^{*}$ and an identity ID $^{*}$ to be challenged to $\mathcal{C}$. The challenge time period $T^{*}$ must differ from any $T$ issued as release queries in Phase $1 . \mathcal{C}$ randomly chooses $b \in\{0,1\}$ and sends a challenge ciphertext $c^{*}=\operatorname{Encrypt}(t p k$, params, $\left.T^{*}, \mathrm{ID}^{*}, m_{b}\right)$ to $\mathcal{A}$.

Phase2

$\mathcal{A}$ can adaptively issue release queries $T$ and decryption queries $(T, I D, c)$ in the same way as Phase1 except that the release query $T$ must differ from the challenge time period $T^{*}$, and the decryption queries $(T, \mathrm{ID}, c)$ must differ from the tuple $\left(T^{*}, \mathrm{ID}{ }^{*}, c^{*}\right)$.

Guess

$\mathcal{A}$ outputs a guess $b^{\prime} \in\{0,1\}$ and wins if $b=b^{\prime}$. 
We define an advantage of $\mathcal{A}$ in the IND-hID-CCA $\mathrm{CR}_{\mathrm{C}}$ game as $\operatorname{Adv}_{\mathcal{T} \mathcal{R} \mathcal{H} \mathcal{I} \mathcal{I} \mathcal{B}, \mathcal{A}}$ INR $(\lambda)=\left|2 \operatorname{Pr}\left[b=b^{\prime}\right]-1\right|$, in which the probability is taken over the random coins used by $\mathcal{C}$ and $\mathcal{A}$. We say that the TRIBE scheme $\mathcal{T} \mathcal{R} \mathcal{H} \mathcal{I} \mathcal{B}$ is IND-hID-CCA ${ }_{C R}$ secure if, for any probabilistic polynomial-time adversary $\mathcal{A}$, the function $A d v_{\mathcal{T} \mathcal{R} \mathcal{H} \mathcal{I B \mathcal { E }}, \mathcal{A}}{ }^{\mathrm{ND}-h \mathrm{D}}(\lambda)$ is negligible in $\lambda$.

\section{CONSTRUCTION OF TRHIBE}

Here we present a generic construction of TRHIBE scheme from two HIBE schemes, and a one-time signature scheme.

\section{A. Construction}

Let $\Pi=$ (HIBE.Setup, HIBE.Ext, HIBE.Del, HIBE.Enc, HIBE.Dec) and $\Pi^{\prime}=\left(\mathrm{HIBE}^{\prime}\right.$.Setup, HIBE'.Ext, HIBE'.Del, $\mathrm{HIBE}^{\prime}$.Enc, HIBE'.Dec) be hierarchical identity-based encryption schemes, and $\Sigma=$ (SigGen, Sign, Verify) be a one-time signature scheme.

A TRHIBE scheme $\Gamma=$ (TS_Setup, KGC_Setup, Release, Extract, Encrypt, Decrypt) is constructed as follows.

Time server setup TS_Setup $(\lambda)$ :

Step 1: Run HIBE.Setup $(\lambda, 1)$ to generate (params, msk). Step 2: Set $t p k=$ params and $t s k=m s k$.

Step 3: Return $(t p k, t s k)$.

Key generation center setup KGC_Setup $(\lambda, \ell)$ :

Step 1: Run $\operatorname{HIBE}^{\prime}$.Setup $(\lambda, \ell)$ to generate (params, msk). Step 2: Return (params, msk).

Release Release $(t p k, t s k, T)$ :

Step 1: Run HIBE.Ext $(t p k, t s k, T)$ to obtain $d_{T}$.

Step 2: Return $d_{T}$.

Extraction Extract $($ params, $m s k$, ID $)$ :

Step 1: Run $\mathrm{HIBE}^{\prime}$. Ext (params, msk, ID) to obtain $d_{\mathrm{ID}_{j}}$.

Step 2: Return $d_{\mathrm{ID}_{j}}$.

Delegate (params, $\left.\mathrm{ID}^{(k)}, d_{\mathrm{ID}^{(k)}}, \mathrm{ID}^{(k+1)}\right)$ :

Step 1: Run $\mathrm{HIBE}^{\prime}$. Del $\left(\right.$ params, $\left.\mathrm{ID}^{(k)}, d_{\mathrm{ID}^{(k)}}, \mathrm{ID}^{(k+1)}\right)$ to obtain $d_{\mathrm{ID}^{(k+1)}}$.

Step 2: Return $d_{\mathrm{ID}(k+1)}$.

Encryption Encrypt $(t p k$, params, $m, T$, ID):

Step 1: Run SigGen $(\lambda)$ to generate $(s k, v k)$.

Step 2: Randomly choose $s_{1} \in\{0,1\}^{|m|}$.

Step 3: Compute $s_{2}=m \oplus s_{1}$.

Step 4: Compute $c_{1}=\operatorname{HIBE}$.Enc $\left(t p k, s_{1} \| v k, T\right)$.

Step 5: Compute $c_{2}=\operatorname{HIBE}^{\prime}$.Enc $\left(\right.$ params, $s_{2} \| v k$, ID $)$.

Step 6: Compute $\sigma=\operatorname{Sign}\left(s k, c_{1} \| c_{2}|| T|| \mathrm{ID}\right)$.

Step 7: Set $c=\left(c_{1}, c_{2}, T\right.$, ID, $\left.v k, \sigma\right)$.

Step 8: Return $c$.

Decryption Decrypt $\left(t p k\right.$, params $\left., c, d_{T}, d_{\mathrm{ID}}\right)$ :

Step 1: Parse c as $c=\left(c_{1}, c_{2}, T\right.$, ID, $\left.v k, \sigma\right)$.

Step 2: If Verify $\left(v k, c_{1}\left\|c_{2}\right\| T|| I \mathrm{D}, \sigma\right)=$ re ject then return

$\perp$ and stop.

Step 3: Compute $s_{1} \| v k^{\prime}=\operatorname{HIBE} \cdot \operatorname{Dec}\left(t p k, c_{1}, d_{T}\right)$.

Step 4: Compute $s_{2} \| v k^{\prime \prime}=\operatorname{HIBE}^{\prime}$.Dec $\left(\right.$ params, $\left.c_{2}, d_{\mathrm{ID}}\right)$.

Step 5: If $v k=v k^{\prime}=v k^{\prime \prime}$ then return $m=s_{1} \oplus s_{2}$, else return $\perp$.

\section{B. Security of TRHIBE.}

1) IND-hID-CCA $A_{T S}$ secure:

Theorem 1: If $\Pi^{\prime}$ is an IND-hID-CCA secure hierarchical identity-based encryption scheme and $\Sigma$ is a OT-sEUFCMA secure one-time signature scheme, then $\Gamma$ is an INDhID-CCA ${ }_{\text {TS }}$ secure timed-release hierarchical identity-based encryption scheme.

Proof(Theorem 1) Suppose $\mathcal{A}$ is an adversary that breaks the IND-hID-CCA $A_{\text {TS }}$ security of $\Gamma$. We construct a simulator $\mathcal{B}$ which breaks the IND-hID-CCA security of the HIBE scheme $\Pi^{\prime}$ using $\mathcal{A}$. Say a ciphertext $c=\left(c_{1}, c_{2}, T, \mathrm{ID}, v k, \sigma\right)$ is valid if Verify $\left(v k, c_{1}\left\|c_{2}\right\| T \| \mathrm{ID}, \sigma\right)=$ accept. Let $c^{*}=$ $\left(c_{1}^{*}, c_{2}^{*}, T^{*}, \mathrm{ID}^{*}, v k^{*}, \sigma^{*}\right)$ be the challenge ciphertext. Let Forge denote the event that $\mathcal{A}$ submits a valid ciphertext $c=\left(c_{1}, c_{2}, T, \mathrm{ID}, v k^{*}, \sigma\right)$ as a decryption query to $\mathcal{C}$ in the Phase2, and Succ denote the event that $\mathcal{B}$ wins the INDhID-CCA game. We prove the following claims.

Claim 1: $\operatorname{Pr}[$ Forge $]$ is negligible.

Claim 2: $\operatorname{Pr}[\operatorname{Succ} \mid \overline{\text { Forge }}]=A d v_{\Gamma, \mathcal{A}}^{\text {IND }}$ ID-CCA $\mathrm{TSS}^{\mathrm{TS}}+\frac{1}{2}$

Proof(Claim 1) We assume Forge occurs. Then, we construct a forger $\mathcal{F}$ who breaks OT-sEUF-CMA security of the onetime signature scheme $\Sigma$, from $\mathcal{A}$. The description of $\mathcal{F}$ is as follows.

Setup

$\mathcal{F}$ receives $v k^{*}$ from $\mathcal{C}$. Then $\mathcal{F}$ runs $(t p k$, $t s k) \leftarrow \operatorname{TS} \operatorname{Setup}(\lambda)$ and (params, msk) $\leftarrow$ $\operatorname{KGC} \operatorname{Setup}(\lambda, \ell) . \mathcal{F}$ sends $t p k, t s k$ and params to $\mathcal{A}$ and keeps msk.

Query

$\mathcal{F}$ can respond to extract queries and decryption queries of $\mathcal{A}$ since $\mathcal{F}$ has tsk and $m s k$. If $\mathcal{A}$ happens to issue a valid ciphertext $c=\left(c_{1}, c_{2}, T\right.$, ID, $\left.v k^{*}, \sigma\right)$ as decryption query to $\mathcal{F}$ before Challenge in the IND-hID-CCA TS game, then $\mathcal{F}$ simply outputs $\left(c_{1}\left\|c_{2}\right\| T|| I D, \sigma\right)$ as forgery and stops.

Challenge

If $\mathcal{A}$ outputs $\left(m_{0}, m_{1}, T^{*}, \mathrm{ID}^{*}\right)$ as challenge, $\mathcal{F}$ randomly chooses $s_{1} \in\{0,1\}^{|m|}$ and $b \in$ $\{0,1\}$, and computes $s_{2}=m_{b} \oplus s_{1}$. Then $\mathcal{F}$ computes $c_{1}^{*}=\operatorname{HIBE} . \operatorname{Enc}\left(t p k, s_{1} \| v k^{*}, T^{*}\right)$ and $c_{2}^{*}=\mathrm{HIBE}^{\prime}$.Enc (params, $\left.s_{2} \| v k^{*}, \mathrm{ID}^{*}\right)$, then issues $m^{*}=\left(c_{1}\left\|c_{2}\right\| T^{*} \| \mathrm{ID}^{*}\right)$ as signing query to $\mathcal{C}$ and obtains $\sigma^{*}$. Finally $\mathcal{F}$ returns $c^{*}=$ $\left(c_{1}^{*}, c_{2}^{*}, T^{*}, \mathrm{ID}^{*}, v k^{*}, \sigma^{*}\right)$ as the challenge ciphertext to $\mathcal{A}$.

Forge

If $\mathcal{A}$ issues a valid ciphertxt $c=\left(c_{1}, c_{2}, T\right.$, ID $\left.v k^{*}, \sigma\right)$ as decryption query, then $\mathcal{F}$ outputs $\left(c_{1}\left\|c_{2}\right\| T|| \mathrm{ID}^{*}, \sigma\right)$ as forgery.

$\mathcal{F}$ can forge the signature if $\mathcal{A}$ issues a decryption query that causes the event Forge. It, however, contradicts that $\Sigma$ is OT-sEUF-CMA secure. Thus, $\operatorname{Pr}[$ Forge $]$ is negligible.

Proof(Claim 2) We construct an adversary $\mathcal{B}$ who breaks IND-hID-CCA security of the HIBE scheme $\Pi^{\prime}$ using $\mathcal{A}$. The description of $\mathcal{B}$ is as follows. 
Setup

$\mathcal{B}$ receives params from $\mathcal{C}$. Then $\mathcal{B}$ runs (tpk, tsk) $\leftarrow$ TS_Setup $(\lambda)$ and sends tpk,tsk and params to $\mathcal{A}$.

Phase1

$\mathcal{B}$ responds to $\mathcal{A}$ 's extraction query ID by issuing ID as $\mathcal{B}$ 's extraction query to $\mathcal{C}$ and obtaining $d_{\mathrm{ID}}$ from $\mathcal{C}$ and returning $d_{\mathrm{ID}}$ to $\mathcal{A}$. $\mathcal{B}$ responds to $\mathcal{A}$ 's decryption query $c$ as follows. If $\operatorname{Verify}\left(v k, c_{1}\left\|c_{2}\right\| T|| \mathrm{ID}, \sigma\right)=$ reject, then $\mathcal{B}$ returns $\perp$ to $\mathcal{A}$. Otherwise $\mathcal{B}$ runs $s_{1} \| v k^{\prime} \leftarrow$ $\operatorname{HIBE} \operatorname{Dec}\left(c_{1}, d_{T}\right)$ and issues decryption query $\left(c_{2}\right.$, ID) to $\mathcal{C}$ and obtains $s_{2} \| v k^{\prime \prime} . \mathcal{B}$ returns $m=$ $s_{1} \oplus s_{2}$ to $\mathcal{A}$ if $v k=v k^{\prime}=v k^{\prime \prime}$, and otherwise Challenge $\mathcal{B}$ returns $\perp$ to $\mathcal{A}$.

If $\mathcal{A}$ outputs $\left(m_{0}, m_{1}, T^{*}, \mathrm{ID}^{*}\right)$ as challenge, $\mathcal{B}$ runs $\left(s k^{*}, v k^{*}\right) \leftarrow \operatorname{SigGen}(\lambda)$ and randomly chooses $s_{1} \in\{0,1\}^{|m|}$ and runs $c_{1}^{*}=$ HIBE.Enc $\left(t p k, r \| v k^{*}, T^{*}\right)$. Then $\mathcal{B}$ computes $M_{0}=\left(m_{0} \oplus r \| v k^{*}\right)$ and $M_{1}=\left(m_{1} \oplus\right.$ $\left.r \| v k^{*}\right)$, and issues $\left(M_{0}, M_{1}, \mathrm{ID}^{*}\right)$ as $\mathcal{B}^{\prime}$ s challenge to $\mathcal{C}$ and obtains cyphertext $c_{2}^{*}$. $\mathcal{B}$ runs $\sigma^{*}=\operatorname{Sign}\left(s k^{*}, c_{1}^{*}\left\|c_{2}^{*}\right\| T^{*} \| \mid \mathrm{ID}^{*}\right)$ and returns $c^{*}=$ $\left(c_{1}^{*}, c_{2}^{*}, T^{*}, \mathrm{ID}^{*}, v k^{*}, \sigma^{*}\right)$ as challenge ciphertext

Phase2 to $\mathcal{A}$.

$\mathcal{B}$ responds to $\mathcal{A}$ 's extraction query ID in the same way as in Phase1. $\mathcal{B}$ responds to $\mathcal{A}$ 's decryption query as follows. The followings are done in a sequential way.

Step1

If $\operatorname{Verify}\left(v k, c_{1}\left\|c_{2}\right\| T\|\mid \mathrm{ID}\|, \sigma\right)=$ reject, then $\mathcal{B}$ returns $\perp$ and skips step2 4 .

Step2

If $v k=v k^{*}$, then $\mathcal{B}$ stops the simulation and outputs a random bit $b^{\prime}$.

Step3

If $\left(c_{2}\right.$, ID $)=\left(c_{2}^{*}\right.$, ID $\left.^{*}\right)$, then $\mathcal{B}$ returns

Step4 $\perp$ and skips step4.

$\mathcal{B}$ responds in the same way as in Phase1.

Guess If $\mathcal{A}$ outputs a bit, then $\mathcal{B}$ outputs a same bit as its guess.

We examine the $\mathcal{B}$ 's simulation of the response to decryption queries in Phase2. In the case of Verify $=$ reject in Step1, $\mathcal{B}$ returns $\perp$ in the same way as in our decryption algorithm, and then it perfectly simulates the challenger in

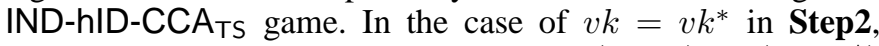
the event Forge occurs. In the case of $\left(c_{2}, \mathrm{ID}\right)=\left(c_{2}^{*}, \mathrm{ID}^{*}\right)$ in Step3, since $c_{2}$ equals to $c_{2}^{*}$, the decryption of $c_{2}$ is $M_{0}=\left(m_{0} \oplus r \| v k^{*}\right)$ or $M_{1}=\left(m_{1} \oplus r \| v k^{*}\right)$. However, since $v k \neq v k^{*}$, the decryption of $c$ is $\perp$, and then $\mathcal{B}$ simulates perfectly. In the case of $\left(c_{2}, \mathrm{ID}\right) \neq\left(c_{2}^{*}, \mathrm{ID}^{*}\right), \mathcal{B}$ can issue the valid decryption query $\left(c_{2}\right.$, ID) to $\mathcal{C}$.

If the event Forge does not occurs, $\mathcal{B}$ perfectly simulates the challengers in the IND-hID-CCA ${ }_{\text {TS }}$ game and wins the IND-hID-CCA game with the same probability that

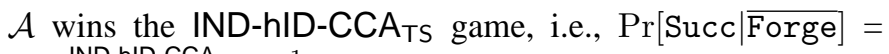
$A d v_{\Gamma, \mathcal{A}}^{\mathrm{IND}-\mathrm{hID}-\mathrm{CCA} \mathrm{AS}_{\mathrm{T}}}+\frac{1}{2}$.

We see that

$$
\begin{aligned}
\operatorname{Pr}[\text { Succ }] & \geq \operatorname{Pr}[\text { Succ } \wedge \overline{\text { Forge }}] \\
& =\operatorname{Pr}[\text { Succ } \mid \overline{\text { Forge }}] \cdot \operatorname{Pr}[\overline{\text { Forge }}] \\
& =\operatorname{Pr}[\text { Succ } \mid \overline{\text { Forge }}] \cdot(1-\operatorname{Pr}[\text { Forge }]) \\
& =\operatorname{Pr}[\text { Succ } \mid \overline{\text { Forge }}]-\operatorname{Pr}[\text { Succ } \mid \overline{\text { Forge }}] \cdot \operatorname{Pr}[\text { Forge }] \\
& \geq \operatorname{Pr}[\text { Succ } \mid \overline{\text { Forge }}]-\operatorname{Pr}[\text { Forge }]
\end{aligned}
$$

then, from Claim 2, we have that

$$
\operatorname{Pr}[\text { Succ }] \geq A d v_{\Gamma, \mathcal{A}}^{\mathrm{IND} \text {-hID-CCA }}{ }^{\mathrm{Ts}}+\frac{1}{2}-\operatorname{Pr}[\text { Forge }] .
$$

If $A d v_{\Gamma, \mathcal{A}}^{\mathrm{IND}-\mathrm{hlD}-\mathrm{CCA}} \mathrm{TS}_{\mathrm{TS}}$ is not negligible, $A d v_{\Pi, \mathcal{B}}^{\mathrm{IND}-\mathrm{hID}-\mathrm{CCA}}=$ $\left|\operatorname{Pr}[\operatorname{Succ}]-\frac{1}{2}\right|$ is not negligible from Claim 1, and it contradicts our assumption. This completes the proof of Theorem 1.

\section{2) IND-hID-CCA CR $_{\text {secure: }}$}

Theorem 2: If $\Pi$ is an IND-hID-CCA secure hierarchical identity-based encryption scheme and $\Sigma$ is a OT-sEUFCMA secure one-time signature scheme, then $\Gamma$ is an IND$\mathrm{hID}-\mathrm{CCA}_{\mathrm{CR}}$ secure timed-release hierarchical identity-based encryption scheme.

Proof(Theorem 2) Suppose $\mathcal{A}$ is an adversary that breaks the IND-hID-CCA TS $_{\text {security of }} \Gamma$. We construct a simulator $\mathcal{B}$ which breaks the IND-hID-CCA security of the HIBE scheme $\Pi$ using $\mathcal{A}$. Say a ciphertext $c=\left(c_{1}, c_{2}, T\right.$, ID, $\left.v k, \sigma\right)$ is valid if $\operatorname{Verify}\left(v k, c_{1} \| c_{2}|| T|| \mathrm{ID}, \sigma\right)=$ accept. Let $c^{*}=$ $\left(c_{1}^{*}, c_{2}^{*}, T^{*}, \mathrm{ID}^{*}, v k^{*}, \sigma^{*}\right)$ be the challenge ciphertext. Let Forge denote the event that $\mathcal{A}$ submits a valid ciphertext $c=\left(c_{1}, c_{2}, T, \mathrm{ID}, v k^{*}, \sigma\right)$ as a decryption query to $\mathcal{C}$ in the Phase2, and Succ denote the event that $\mathcal{B}$ wins the INDhID-CCA game. We prove the following claims.

Claim 3: $\operatorname{Pr}[$ Forge $]$ is negligible.

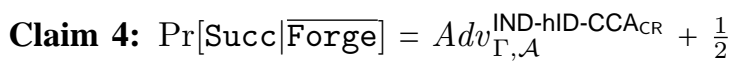

Proof(Claim 3) We assume Forge occurs. Then, We construct a forger $\mathcal{F}$ who breaks OT-sEUF-CMA security of the onetime signature scheme $\Sigma$, from $\mathcal{A}$. The description of $\mathcal{F}$ is as follows.

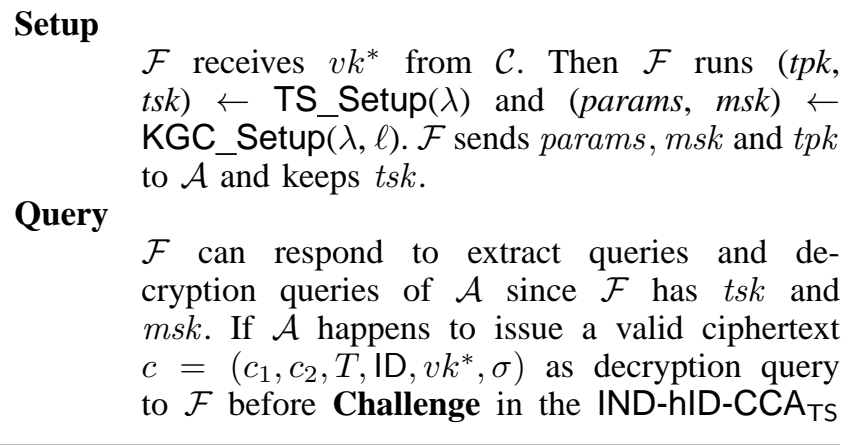


Challenge

game, then $\mathcal{F}$ simply outputs $\left(c_{1}|| c_{2} \| T|| \mathrm{ID}, \sigma\right)$ as forgery and stops.

If $\mathcal{A}$ outputs $\left(m_{0}, m_{1}, T^{*}, \mathrm{ID}^{*}\right)$ as challenge , $\mathcal{F}$ randomly chooses $s_{1} \in\{0,1\}^{|m|}$ and $b \in$ $\{0,1\}$, and computes $s_{2}=m_{b} \oplus s_{1}$. Then $\mathcal{F}$ computes $c_{1}^{*}=\operatorname{HIBE} \operatorname{Enc}\left(t p k, s_{1} \| v k^{*}, T^{*}\right)$ and $c_{2}^{*}=\mathrm{HIBE}^{\prime} \cdot \operatorname{Enc}\left(\right.$ params $\left., s_{2} \| v k^{*}, \mathrm{ID}^{*}\right)$, then issues $m^{*}=\left(c_{1}\left\|c_{2}\right\| T^{*} \| \mathrm{ID}^{*}\right)$ as signing query to $\mathcal{C}$ and obtains $\sigma^{*}$. Finally $\mathcal{F}$ returns $c^{*}=$ $\left(c_{1}^{*}, c_{2}^{*}, T^{*}, \mathrm{ID}^{*}, v k^{*}, \sigma^{*}\right)$ as the challenge ciphertext to $\mathcal{A}$

Forge

If $\mathcal{A}$ issues a valid ciphertxt $c=$ $\left(c_{1}, c_{2}, T, \mathrm{ID}, v k^{*}, \sigma\right)$ as decryption query, then $\mathcal{F}$ outputs $\left(c_{1}\left\|c_{2}\right\| T|| \mathrm{ID}^{*}, \sigma\right)$ as forgery.

$\mathcal{F}$ can forge the signature if $\mathcal{A}$ issues a decryption query that causes the event Forge. It, however, contradicts that $\Sigma$ is OT-sEUF-CMA secure. Thus, $\operatorname{Pr}[$ Forge $]$ is negligible.

Proof(Claim 4) We construct an adversary $\mathcal{B}$ who breaks IND-hID-CCA security of the HIBE scheme $\Pi$ using $\mathcal{A}$. The description of $\mathcal{B}$ is as follows.

Setup

$\mathcal{B}$ receives params from $\mathcal{C}$. We call this params tpk. Then $\mathcal{B}$ runs (params, msk) $\leftarrow$ $\operatorname{KGC} \operatorname{Setup}(\lambda, \ell)$ and sends params, msk and tpk to $\mathcal{A}$.

Phase1

$\mathcal{B}$ responds to $\mathcal{A}$ 's release query $T$ by issuing $T$ as $\mathcal{B}$ 's extraction query to $\mathcal{C}$ and obtaining $d_{T}$ from $\mathcal{C}$ and returning $d_{T}$ to $\mathcal{A}$. $\mathcal{B}$ responds to $\mathcal{A}$ 's decryption query $c$ as follows. If $\operatorname{Verify}\left(v k, c_{1} \| c_{2}|| T|| \mathrm{ID}, \sigma\right)=$ reject, then $\mathcal{B}$ returns $\perp$ to $\mathcal{A}$. Otherwise $\mathcal{B}$ runs $s_{2} \| v k^{\prime} \leftarrow$ HIBE.Dec $\left(c_{2}, d_{\mathrm{ID}}\right)$ and issues decryption query $\left(c_{1}, T\right)$ to $\mathcal{C}$ and obtains $s_{1} \| v k^{\prime \prime} . \mathcal{B}$ returns $m=$ $s_{1} \oplus s_{2}$ to $\mathcal{A}$ if $v k=v k^{\prime}=v k^{\prime \prime}$, and otherwise

Challenge $\mathcal{B}$ returns $\perp$ to $\mathcal{A}$.

If $\mathcal{A}$ outputs $\left(m_{0}, m_{1}, T^{*}, \mathrm{ID}^{*}\right)$ as challenge, $\mathcal{B}$ runs $\left(s k^{*}, v k^{*}\right) \leftarrow \operatorname{SigGen}(\lambda)$ and randomly chooses $s_{1} \in\{0,1\}^{|m|}$ and runs $c_{1}^{*}=$ HIBE.Enc (params, $\left.r \| v k^{*}, \mathrm{ID}^{*}\right)$. Then $\mathcal{B}$ computes $M_{0}=\left(m_{0} \oplus r \| v k^{*}\right)$ and $M_{1}=\left(m_{1} \oplus\right.$ $\left.r \| v k^{*}\right)$, and issues $\left(M_{0}, M_{1}, T^{*}\right)$ as $\mathcal{B}$ 's challenge to $\mathcal{C}$ and obtains cyphertext $c_{2}^{*}$. $\mathcal{B}$ runs $\sigma^{*}=\operatorname{Sign}\left(s k^{*}, c_{1}^{*}\left\|c_{2}^{*}\right\| T^{*} \| \mid \mathrm{ID}^{*}\right)$ and returns $c^{*}=$ $\left(c_{1}^{*}, c_{2}^{*}, T^{*}, \mathrm{ID}^{*}, v k^{*}, \sigma^{*}\right)$ as challenge ciphertext to $\mathcal{A}$.

Phase2

$\mathcal{B}$ responds to $\mathcal{A}$ 's extraction query $T$ in the same way as in Phase1. $\mathcal{B}$ responds to $\mathcal{A}$ 's decryption query as follows. The followings are done in a sequential way.

Step1

If $\operatorname{Verify}\left(v k, c_{1}\left\|c_{2}\right\| T\|\mid \mathrm{ID}\|, \sigma\right)=$ reject, then $\mathcal{B}$ returns $\perp$ and skips step2 4 .

\begin{abstract}
Step2
If $v k=v k^{*}$, then $\mathcal{B}$ stops the simulation and outputs a random bit $b^{\prime}$.

Step3

If $\left(c_{1}, T\right)=\left(c_{1}^{*}, T^{*}\right)$, then $\mathcal{B}$ returns

Step4 $\perp$ and skips step4.

$\mathcal{B}$ responds in the same way as in Phase1.
\end{abstract}

Guess

If $\mathcal{A}$ outputs a bit, then $\mathcal{B}$ outputs a same bit as its guess.

We examine the $\mathcal{B}$ 's simulation of the response to decryption queries in Phase2. In the case of Verify $=$ reject in Step1, $\mathcal{B}$ returns $\perp$ in the same way as in our decryption algorithm, and then it perfectly simulates the challenger in IND-hID-CCA TS game. In the case of $v k=v k^{*}$ in Step2, the event Forge occurs. In the case of $\left(c_{1}, T\right)=\left(c_{1}^{*}, T^{*}\right)$ in Step3, since $c_{1}$ equals to $c_{1}^{*}$, the decryption of $c_{1}$ is $M_{0}=\left(m_{0} \oplus r \| v k^{*}\right)$ or $M_{1}=\left(m_{1} \oplus r \| v k^{*}\right)$. However, since $v k \neq v k^{*}$, the decryption of $c$ is $\perp$, and then $\mathcal{B}$ simulates perfectly. In the case of $\left(c_{1}, T\right) \neq\left(c_{1}^{*}, T^{*}\right), \mathcal{B}$ can issue the valid decryption query $\left(c_{1}, T\right)$ to $\mathcal{C}$.

If the event Forge does not occurs, $\mathcal{B}$ perfectly simulates the challengers in the IND-hID-CCA $A_{C R}$ game and wins the IND-hID-CCA game with the same probability that $\mathcal{A}$ wins the IND-hID-CCA ${ }_{C R}$ game, i.e., $\operatorname{Pr}[$ Succ $\mid \overline{\text { Forge }}]=$

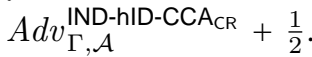

We see that

$$
\begin{aligned}
\operatorname{Pr}[\text { Succ }] & \geq \operatorname{Pr}[\text { Succ } \wedge \overline{\text { Forge }}] \\
& =\operatorname{Pr}[\text { Succ } \mid \overline{\text { Forge }}] \cdot \operatorname{Pr}[\text { Forge }] \\
& =\operatorname{Pr}[\text { Succ } \mid \overline{\text { Forge }}] \cdot(1-\operatorname{Pr}[\text { Forge }]) \\
& =\operatorname{Pr}[\text { Succ } \mid \overline{\text { Forge }}]-\operatorname{Pr}[\text { Succ } \mid \overline{\text { Forge }}] \cdot \operatorname{Pr}[\text { Forge }] \\
& \geq \operatorname{Pr}[\text { Succ } \mid \overline{\text { Forge }}]-\operatorname{Pr}[\text { Forge }]
\end{aligned}
$$

then, from Claim 3, we have that

$$
\operatorname{Pr}[\text { Succ }] \geq A d v_{\Gamma, \mathcal{A}}^{\mathrm{IND}-\mathrm{hID}-\mathrm{CCA} \mathrm{AR}}+\frac{1}{2}-\operatorname{Pr}[\text { Forge }] .
$$

If $A d v_{\Gamma, \mathcal{A}}^{\mathrm{IND}-\mathrm{hlD}-\mathrm{CCA} \mathrm{AR}_{\mathrm{CR}}}$ is not negligible, $A d v_{\Pi, \mathcal{B}}^{\mathrm{IND}-\mathrm{hID}-\mathrm{CCA}}=$ $\left|\operatorname{Pr}[\operatorname{Succ}]-\frac{1}{2}\right|$ is not negligible from Claim 4, and it contradicts our assumption. This completes the proof of Theorem 2.

\section{CONCLUSION}

In this paper, we introduced a notion of TRHIBE and defined IND-hID-CCA $\mathrm{CR}_{\mathrm{C}}$ security and IND-hID-CCA $\mathrm{TS}_{\mathrm{TS}}$ security. Moreover, we showed a generic construction of TRHIBE in which a constructed scheme achieves those security if the primitive HIBE schemes are IND-hID-CCA secure and the primitive one-time signature scheme is OT-sEUF-CMA secure.

\section{ACKNOWLEDGMENT}

This work was supported by JSPS KAKENHI Grant Number 26330160 . 


\section{REFERENCES}

[1] T. May, "Timed-release crypto," Manuscript, February 1993.

[2] A. C.-F. Chan and I. F. Blake, "Scalable, server-passive, useranonymous timed release cryptography," in ICDCS 2005. IEEE Computer Society, 2005, pp. 504-513.

[3] J. H. Cheon, N. Hopper, Y. Kim, and I. Osipkov, "Timed-release and key-insulated public key encryption," in FC 2006, ser. Lecture Notes in Computer Science, G. Di Crescenzo and A. Rubin, Eds., vol. 4107. Springer-Verlag, 2006, pp. 191-205.

[4] - "Provably secure timed-release public key encryption," ACM Transactions on Information and System Security (TISSEC), vol. 11, no. 2, p. Article 4, 2008.

[5] J. Cathalo, B. Libert, and J.-J. Quisquater, "Efficient and non-interactive timed-release encryption," in ICICS 2005, ser. Lecture Notes in Computer Science, S. Qing, W. Mao, J. Lopez, and G. Wang, Eds., vol. 3783. Springer-Verlag, 2005, pp. 291-303.

[6] T. Oshikiri and T. Saito, "Timed-release identity-based encryption," IPSJ Journal, vol. 55, no. 9, pp. 1964-1970, sep 2014.

[7] Y. Dodis and J. Katz, "Chosen-ciphertext security of multiple encryption," in TCC 2005, ser. Lecture Notes in Computer Science, J. Kilian, Ed., vol. 3378. Springer-Verlag, 2005, pp. 188-209.

[8] D. Boneh, X. Boyen, and E.-J. Goh, "Hierarchical identity based encryption with constant size ciphertext," in Proceedings of the 24th Annual International Conference on Theory and Applications of Cryptographic Techniques, ser. EUROCRYPT'05. Berlin, Heidelberg: Springer-Verlag, 2005, pp. 440-456.

[9] A. Lewko and B. Waters, "New techniques for dual system encryption and fully secure hibe with short ciphertexts," in Theory of Cryptography, ser. Lecture Notes in Computer Science, D. Micciancio, Ed. Springer Berlin Heidelberg, 2010, vol. 5978, pp. 455-479.

[10] R. C. Merkle, "A digital signature based on a conventional encryption function," in A Conference on the Theory and Applications of Cryptographic Techniques on Advances in Cryptology, ser. CRYPTO '87. London, UK, UK: Springer-Verlag, 1988, pp. 369-378. [Online]. Available: http://dl.acm.org/citation.cfm?id=646752.704751 